\title{
1940 - 1944 : « On meurt de soif » mais les eaux sont mortes. La portée symbolique de l'eau chez les écrivains français sous l'Occupation
}

\section{Saiz-Cerreda, María Pilar}

Universidad de Navarra, mpsaiz@unav.es

\begin{abstract}
Resumen
Cuando en 1940 Francia se convierte en territorio ocupado, las condiciones de vida son tan penosas que los franceses tienen grandes dificultades para sobrevivir. Sin embargo, por muy espantosas que sean las condiciones materiales, nada se puede comparar a la degradación de la vida del Espíritu, a la ausencia de lo espiritual. Los franceses pasan hambre, frío, pero peor aún, su espíritu muere. Los franceses van perdiendo su identidad. "El alma está tan yerma hoy. Morimos de sed», exclamaba Antoine de SaintExupéry en una carta a su madre en 1940 (1994: 83)1. Y sin embargo no podemos calmar nuestra sed porque solo existe « esta ciénaga » (Saint-Exupéry, $1994: 310)^{2}$ El dolor es demasiado fuerte porque es el patrimonio espiritual francés el que está en peligro y numerosos escritores son conscientes de ello. La esencia de la vida, el agua clara, límpida, el agua que da la vida, se ha transformado en agua de muerte, " un agua que debe ensombrecerse, un agua que va a absorber el oscuro sufrimiento. Toda agua viva es un agua destinada a volverse lenta, pesada. Toda agua viva es un agua a punto de morir » (Bachelard, 1942 : 65-66). La vida queda ahogada como explica Bachelard (1942: 65) y solo existen aguas estancadas, «aguas muertas» $(1942: 63)^{3}$. En esta comunicación quiero mostrar cómo sienten y manifiestan los escritores esta crisis, esta pérdida de identidad, esta muerte ; y cómo proponen la escritura como remedio, como vía de salvación, gracias en especial a la acción autobiográfica. La escritura se convertirá en el único lugar en que poder calmar la sed.
\end{abstract}

Palabras clave : agua estancada ; muerte ; identidad ; escritura autobiográfica ; diarios

\section{Résumé}

Quand en 1940 la France devient territoire occupé, les conditions de vie se dégradent à tel point que les Français vivent en grande difficulté. Pourtant, aussi épouvantables que les conditions matérielles de vie soient-elles, rien n'est comparable à la dégradation de la vie de l'Esprit, au manque du spirituel. Les Français sont affamés, les Français ont froid, mais pire encore, les Français meurent dans l'esprit. Les Français perdent peu à peu leur identité. "C'est l'âme aujourd'hui qui est tellement déserte. On meurt de soif » (1994 : 83), s'exclamait Antoine de Saint-Exupéry dans une lettre à sa mère en 1940. Et pourtant on ne peut pas s'abreuver parce qu'il n'existe que «ce marécage » (Saint-Exupéry, 1994 : 310). La douleur est trop forte parce que c'est le patrimoine spirituel français qui est en péril et de nombreux écrivains en sont très conscients. L'essence de la vie, l'eau claire, l'eau limpide, l'eau qui donne la vie, s'est transformée en eau de mort, " une eau qui doit s'assombrir, une eau qui va absorber la noire souffrance. Toute eau vive est une eau dont le destin est de s'alentir, de s'alourdir. Toute eau vivante est une eau qui est sur le point de mourir» (Bachelard, 1942 : 65-66). La vie est étouffée comme explique Bachelard (1942 : 65) et il n'y a que des eaux stagnantes, des « eaux mortes » (1942: 63). Dans cette communication je voudrais montrer comment les écrivains ressentent et manifestent cette crise, cette perte d'identité, cette mort ; et comment l'écriture est proposée comme remède, comme voie de salut grâce à la démarche autobiographique d'une manière spéciale. L'écriture deviendra le seul endroit où aller pour s'abreuver.

\footnotetext{
${ }^{1}$ Traduit par l'auteur de l'article.

${ }^{2}$ Traduit par l'auteur de l'article.

${ }^{3}$ Traduit par l'auteur de l'article.
} 
1940-1944: “On meurt de soif» mais les eaux sont mortes. La portée symbolique de l'eau chez les écrivains français sous l'Occupation

Mots-clés : eaux stagnantes ; mort ; identité ; écriture autobiographique ; journaux

\begin{abstract}
In 1940 occupied France, living conditions are so bad that the French find it difficult to survive. Nevertheless, however dreadful their material conditions, nothing can compare with the degradation of the soul's life, the absence of the spiritual. The French suffer from hunger and cold, but, worse still, their soul dies. They lose their identity little by little. "The soul is today so void. We die of thirst ", exclaimed Antoine de Saint-Exupéry in a letter to his mother dated 1940 (1994: 83). Nevertheless, we cannot quench our thirst because there is only «this cesspool » (Saint-Exupéry, $1994: 310)$. The pain is unbearable since it is the French spiritual patrimony that is at stake, a situation noted by numerous writers. The essence of life, the clear, clean water that grants life, turns into water of death, " a water that needs to go somber, a water that will absorb the dark suffering. All running water is destined to become slow, heavy. All running water is water about to die " (Bachelard, $1942:$ 65-66). Life is choked, as explains Bachelard (1942:65), and there are only stagnant waters, «dead waters » (1942: 63). In this paper I aim to show how writers feel and manifest this crisis, this loss of identity, this death; and how they propose writing as a means of healing, as a way to salvation, particularly thanks to the autobiographical action. Writing will be the only place where they can quench their thirst.
\end{abstract}

Keywords : stagnant water ; death ; identity ; autobiographical writing ; diaries

Gaston Bachelard expliquait dans son livre L'eau et les rêves comment l'eau est un élément associé à l'imaginaire individuel et collectif de toutes les sociétés, qui se transforme en métaphore des signifiés profonds transcendant les pures apparences au-delà de l'expérience sensible. On pourra le constater dans cette communication. La période comprise entre 1940 et 1944 est restée «synonyme de catastrophe sans précédent » (Bédarida, 1977 : 13). Dans la France occupée, les conditions de vie se dégradent à tel point que les Français vivent en grande difficulté. Les Français sont affamés, les Français ont froid, mais pire encore, les Français meurent dans l'esprit. Les Français perdent peu à peu leur identité. La vie est étouffée, comme explique Bachelard (1942 : 65) et il n’y a que des eaux stagnantes, des « eaux mortes » (1942 : 63). Je voudrais montrer ici comment quatre écrivains non collaborationnistes ressentent et exposent cette perte d'identité, cette mort, dans leurs journaux : Julien Green, avec la partie de son journal correspondant aux années 1940-1944 ; Henri Thomas, et les pages de son journal recouvrant le même créneau temporel ; Léon Werth, avec son journal Déposition et Jean Guéhenno, avec son Journal des années noires. Je prendrai comme point de départ les images relevant des eaux stagnantes et des eaux mortes pour analyser la portée de la signification profonde qui en découle.

Dans notre tradition culturelle occidentale, l'eau peut être contemplée de deux versants opposés et complémentaires à la fois : d'un côté, le versant positif qui envisage l'eau comme source de vie, comme signe de "fertilisation », de « bénédiction », (Chevalier et Gheerbrandt, 1982 : 376), un don de la divinité. C'est «l'eau vive », l'eau pure qui « introduit dans l'éternel » (Chevalier et Gheerbrandt, 1982 : 377). Par contre, si l'on se situe sur le versant négatif, l'eau devient source de mort, de destruction. L'eau n'est plus pure et la mort sera d'autant plus présente que sur celle-là s'est opérée un pervertissement, l'eau s'est mélangée à la terre adoptant la forme d'un marais, d'un marécage, d'une mare. Si l'eau pure conduisait à l'éternel, l'eau stagnante conduit inévitablement à la mort. Cette eau est le symbole de tout ce qui a le pouvoir de tuer cette vie : l’anéantissement, le vide, le désert...

De toute évidence l'Occupation ne saurait être identifiée ou définie que par des mots ou des expressions faisant allusion aux eaux stagnantes, aux eaux de mort. Pourtant la lecture des journaux offre des résultats apparemment contradictoires. Les mots « marais », « mare » ou « marécage » ont à peine laissé une trace physique dans l'écriture, à quelques exceptions près.

À vrai dire, la présence de ces eaux mortes dans les journaux qui nous occupent se laisse sentir et voir constamment si bien les diaristes préfèrent éviter le mot symbolique. Il se produit un transfert ou un déplacement de ce mot contenant «des connotations potentielles déjà existantes» (Darwish, 2011: 158), à l'expression des «effets associatifs et émotionnels » (Ricœur, 1975 : 300) que chaque auteur a accordé au mot. La réalité de la guerre s'empreint ainsi de nouvelles et plus riches significations. Les expressions utilisées désormais contribuent à la construction d'un sens plus 
profond des contenus puisqu’elles sont régies par ce que Ricœur, a dénommé «principe de la plénitude » (1972 : 105). C’est-à-dire, dans la mesure où les diaristes subissent, assimilent et intériorisent leurs expériences de la guerre et de l'Occupation, la projection qu'ils en feront dans les journaux atteindra une plénitude de signification qui sera mise en évidence par des expressions suggestives dont la portée dépassera le sens traditionnel toujours associé au mot symbolique.

Or, ce transfert du mot aux effets est réalisé par étapes. Ainsi donc, une première étape serait marquée par les données empiriques, par la contemplation des conditions de vie sous l’Occupation qui s'empirent avec le temps. Pour autant, le constat de la faim, du froid n’est jamais dépourvu de subjectivité. Bien au contraire, les auteurs en souffrent des conséquences, intériorisent cette expérience et ne peuvent pas se résigner à un message neutre :

\begin{abstract}
Je passe des heures la tête dans mes mains - s'exclamait déjà Guéhenno au début de l'Occupation - , dans une étrange position, celle du pays lui-même peut-être [...] Hier, j'ai fait la queue cinq heures durant, à la mairie, pour avoir nos cartes d'alimentation. J'écoutais les gens. Mais les gens ont la tête aussi vide que le ventre. La confusion des esprits est effroyable. La foule est sans espérance, résignée (Guéhenno, 1947 : 48).
\end{abstract}

Guéhenno n’a pas intérêt à élaborer un discours « objectif » éloigné d’implications personnelles. C’est pourquoi il écrit par détournements et associations de sens. Il remplace le mot « faim » pour aller au-delà et montrer à quel point les conditions de vie sont devenues graves. En même temps il réalise une association importante car il ne veut pas rester attaché au plan physique ou matériel, mais arriver au plan spirituel : le manque d’espérance. Un an plus tard, ce manque d’espérance s’est transformé en désespoir et Guéhenno est tourmenté par le constat de la dégradation des esprits : « Dans ce quartier “communard” où j’habite, entre la rue Haxo et la rue des Rosiers, le petit peuple longtemps résigné tombe au désespoir. Il n’y a plus rien à manger. Toute la viande a été saisie depuis quinze jours » (1947 : 205). C’est la dignité des hommes qui est en jeu et de plus en plus, ces diaristes vont mettre en correspondance les conséquences terribles de la faim, c’est-à-dire, une probable mort du corps, avec les conséquences plus terribles encore d'une faim d’ordre supérieur, la mort spirituelle. Ces mots de Werth ne laissent pas de doute :

Il en est de la solitude comme de la faim. Qui est resté plusieurs jours sans manger ne digère pas une trop grossière nourriture. J'ai rencontré G... qui vient de prendre ses vacances à quelques kilomètres d'ici. Tout se traduit dans sa tête en formules algébriques. Les événements passent sur lui comme sur une toile cirée. Il n’a d’angoisse qu’en ce qui concerne le ravitaillement et il ne voit dans l'avenir d'autre menace que celle d'une famine (1992 : 233$)$.

La réalité s’impose aux diaristes et on commence à apprécier les premiers signes d'un procès de symbiose entre l'événement extérieur et l'existence individuelle. L’événement s’intériorise davantage et dans ce mouvement vers le dedans du diariste, celui-là provoque des effets qui vont devenir par la suite des «noyaux de personnalité » (Mauron, 1962 : 210) contribuant à définir dorénavant le rôle et la fonction de chaque journal et le rôle, la fonction, la mission, bref, l'identité du diariste par ce temps de guerre.

On arrive sans solution de continuité à la deuxième étape de ce transfert du mot symbolique aux effets. L’influence des eaux mortes continue en un crescendo qui n'a pas de limite. Le sort de la France marquera le sort de chaque individu et vice-versa, chaque destin individuel sera le paradigme de ce en quoi la France est en train de se convertir, l'espace de « la pourriture de l’homme » (Werth, 1992 : 185) où il n’existe que mépris, honte, silence, méfiance, peur, horreur, haine, néant, vide, douleur, angoisse, dissolution, anéantissement ; en un mot, la mort. Et à cause de ce procès de symbiose entre l'événement et les existences individuelles, on arrive à une identification entre la réalité de l’individu et la réalité de la France. Le diariste se trouve alors « menacé, et tout penché déjà vers sa disparition » (Richard, 1964 : 29).

C’est le début de «[...] la complète stagnation psychique, l’âme morte », tel que Paul Diel conclut (Chevalier et Gheerbrant, 1982 : 381). C’est la perte d'identité aussi bien individuelle que collective.

Dans ces conditions il ne reste qu'une seule chose à faire pour les auteurs : se retourner vers soi et écrire le journal dans le but de reconstruire leur identité et de ce fait, de reconstruire l’identité, réduite à néant, de la France. Le journal est 
1940-1944: “On meurt de soif» mais les eaux sont mortes. La portée symbolique de l'eau chez les écrivains français sous l'Occupation

considéré le seul espace de liberté et d’indépendance voire de résistance, le refuge personnel contre l’anéantissement, la perte d'identité et la dissolution du moi. C’est donc le genre qui affirme le triomphe de la vie sur la mort.

Pour Werth, par exemple, le fait de tenir un journal est un acte de témoignage parce qu’il ne faut pas oublier la guerre et ses atrocités. De cette manière, il devient non seulement un témoin privilégié qui ne se laisse pas détruire, mais un «bon témoin », qui peut rapporter les «acta, [les] cogitata et [les] sentita » (Simonet-Tenant, 2004 : 115). D’une manière spéciale, sa conscience d'appartenance au peuple juif, un aspect auquel il s’était jusqu'alors montré indifférent : «Quelle lâcheté serait de délibérer sur le point de savoir si je me sens ou je ne me sens pas juif ! [...] je suis juif » (Werth, 1992 : 226).

L’identité narrative relevant de l'écriture du journal de Werth est celle d’un écrivain juif, patriote, sensible à la souffrance du peuple et porteur des souffrances de son peuple. Et aussi celle d’un homme libre malgré les circonstances, qui écrit comme " acte d’engagement » (Heuré, 2006 : 260-261) pour ne jamais oublier et pour témoigner donc " par “devoir et fidélité” à des compagnons morts, emprisonnés, disparus, afin que le monde sache - car tout aurait pu échouer » (Steel, 1991 : 11-12).

Le cas de Guéhenno apporte une autre perspective du rôle du diariste et de son journal, car le but de son écriture vise avant tout à « rejoindre les choses éternelles » (1947: 15). Il est évident que son journal cherche " une dimension de transcendance » (Le Bris, $2010: 16$ ) d'autant plus que l’Occupation devient oppressante.

Lorsqu'il contemple la réalité quotidienne, il se sent « plein de douleur, de colère et de honte » (1947 : 15). La France se trouve toute souillée par «l'invasion des rats » (1947: 14). Il ne peut pas rester impassible et il décide de résister se servant des armes de l'écriture et de la pensée (1947 : 75). C’est pourquoi il commence à tenir son journal dans le but de créer un espace de liberté où il pourra se construire son identité : il est surtout et par-dessus tout bon Français et écrivain Français. Or un bon Français est, ajoute Steel, celui « qui parle la langue et partage les valeurs de la culture française » (1991 : 35). En effet, se tenant fidèle à ces principes, il pourra sauver son esprit, et de cette façon il sauvera l'esprit de la France.

Pour lui, il n’existe qu'un chemin pour y parvenir : le silence, un silence qui a un côté positif, celui de la création de l'écriture. C’est le silence qui «affirme [...] la victoire positive sur la mort » (Mauron, 1962 : 233). Et aussi, il y a un silence négatif, le silence imposé de l'extérieur, celui de la contrainte qui l’oblige à se taire « tout ce que je pense » (16). Ou bien, un silence imposé par lui-même parce que « je ne veux rien écrire ici de ces hommes gris » (Guéhenno, 1947 : 15). S'il ne peut pas parler ou à la rigueur, s’il ne veut pas parler d’une réalité qu'il n’assume pas, il se servira de l'écriture pour mettre en lieu sûr tout ce qui a trait à un bon Français, tout ce qui le rend libre : les valeurs incarnées par les grands penseurs de la France, sa culture et son humanisme de siècles, qui ont été les responsables du rayonnement spirituel de la France partout dans le monde.

Pour Green, l'enjeu de sa démarche d'écriture ne pourrait être saisi sans mettre l'accent sur une circonstance vitale et existentielle décisive qui va marquer le cap de sa vie durant les années de l’Occupation : l'exil, qui l’oblige à se définir vite, les premiers jours passés aux Etats-Unis : « Je suis écrivain français et ne peux plus vivre en France » (1975 : 519$)$. Dans ces circonstances, écrire un journal consiste spécialement à trouver et à « en fixer les points de repère » (Green, 1973 : 1399), et éviter ainsi « l'effroi de disparaître tout entier, oui, quelque chose d'apparenté à la peur de mourir » (Green, 1973 : 1399) parce que « Si je ne suis pas écrivain français, que suis-je donc ? » (Green, 1975 : 543 ).

Or, si ses points de repère sont l'écriture et sa patrie, l'exil devient une menace pour son identité. Il se voit complètement désorienté dans un autre pays, avec une autre langue qu'il doit adopter et qui n’est pas la sienne à proprement parler : « Il faut, par conséquent, que j'écrive désormais en anglais » (Green, 1975 : 519) avec les risques de perte identitaire qu’il encourt : « ma vraie personnalité ne peut guère s’exprimer qu’en français » (Green, 1975 : 807). Il se sent donc « dépaysé et pas du tout à ma place » (Green, 1975 : 522) ; humilié, triste et en colère, parfois même désespéré, il n’a d’autre solution, s'il veut conserver ses repères, que de se mettre en route « vers ce royaume intérieur où les armées totalitaires ne nous atteindront jamais » (Green, 1975 : 525). Dans ce sens, c’est la fidélité à son journal qui le rachète de l’anéantissement et qui le tient en vie car, chez lui, « écrire et être demeurent étroitement confondus » (Saint-Jean, 1975 : XVI).

Thomas, enfin, réalise la démarche diaristique en vue de « savoir qui il est » (2008 : 354). S’il parvient à se sauver par l'écriture et dans l'écriture, il est à même de défricher le chemin pour le salut du peuple. En effet, le péril qui surplombe tout lui fait découvrir l'identification existante entre lui et le peuple : «Dans ce que l'avenir semble me présenter de 
menaçant, il me faut distinguer [...] ce qui va contre mon idée de la beauté et de la justice ; contre mon idée du bonheur ; contre ma vie elle-même, sa racine confondue avec celle de tout un peuple » (Thomas, 2008 : 302). La menace la plus grave étant l'écrasement de l'esprit, il se doit d'entreprendre une lutte contre tout ce qui est susceptible de détruire l'esprit. Il n'existe en fait pour lui une autre manière de le faire que de « retrouver l'état créateur » (Thomas, 2008 : 290). Avoir recours à l'écriture, écrire sans arrêt et refuser «la réalité extérieure, royaume du chaos » (Saiz-Cerreda, 2010 : 232). Seulement ainsi on obtiendra un « degré de civilisation personnelle qui [...] peut former dans le peuple un élément plus capable que tous les partisans réunis de défendre réellement ce qui fait la France » (Thomas, 2008 : 299). Il s’agit pour lui de vivre pour et dans une réalité intérieure, mais aussi il s’agit de montrer aux autres que c'est la seule manière de vivre : vivre dans l'esprit. Sa vie n’a donc qu’un but : « rendre un rayon d'espérance à une société anéantie, maintenir une présence spirituelle, créatrice, au-dessus de la foule abrutie » (Saiz-Cerreda, 2010 : 233). Agissant de la sorte il essaie de tracer la voie de salut pour lui et la France.

Werth, Guéhenno, Green et Thomas. Quatre écrivains d’une grande lucidité qui découvrent que leur mission consiste à « porter hardiment témoignage des valeurs spirituelles » (Bédarida, 2001 : 9) pour lutter contre les eaux stagnantes qui envahissent et imprègnent la réalité de la France de l’Occupation. Bref, nous pouvons conclure avec Bédarida, que la mission de chacun d'eux « était de travailler à restaurer l'humain dans un univers de négation de l'homme » (Bédarida, 2001 : 10). Et ce faisant, ils peuvent construire ou reconstruire une identité menacée : l’identité individuelle qui est aussi l’identité de la France, en définitive.

\section{Références bibliographiques}

BACHELARD, Gaston (1942). L'eau et les rêves. Paris : José Corti.

BEDARIDA, François et BEDARIDA, Renée (éds.) (2001). La Résistance spirituelle 1941-1944. Les Cahiers clandestins du Témoignage chrétien. Paris : Albin Michel.

BEDARIDA, Renée, Témoignage chrétien 1941-1944 (1977). Les Armes de l'Esprit. Paris : Les Éditions ouvrières.

Chevalier, Jean et Gheerbrandt, Alain (1982). Dictionnaire des symboles. Mythes, rêves, coutumes, gestes, formes, figures, couleurs, nombres. Paris : Robert Laffont / Jupiter.

DARWISH, Housamedden (2011). Paul Ricoeur et la problématique de la méthode dans l'herméneutique. Interpréter, comprendre et expliquer dans les théories du symbole, du texte, de la métaphore et du récit. Paris : L’Harmattan.

GreEn, Julien (1973). « Tenir un journal » dans Green, Julien. Euvres complètes. Paris : Gallimard. T. III, p. 1399-1430.

GreEN, Julien (1975). Euvres complètes. Paris : Gallimard. T. IV.

GuEHENNO, Jean (1947). Journal des années noires 1940-1944. Paris : Gallimard.

HeuRE, Gilles (2006). L’insoumis Léon Werth 1878-1955. Paris : Viviane Hamy.

Le Bris, Michel et Rouaud, Jean (dirs.) (2010). Je est un autre. Pour une identité-monde. Paris : Gallimard.

Mauron, Charles (1962). Des métaphores obsédantes au mythe personnel. Introduction à la Psychocritique. Paris : José Corti.

RiCHARD, Jean-Pierre (1964). Onze études sur la poésie moderne. Paris : Éditions du Seuil.

RICOEuR, Paul (1972). « La métaphore et le problème central de l’herméneutique » dans Revue philosophique de Louvain. Éd. de l’Institut Supérieur de Philosophie, Février 1972, quatrième série, t. 70, nº 5, p. 93-112.

SAINT-EXUPERY, Antoine de (1994). Écrits de guerre. 1939-1944. Paris : Gallimard.

SAINT-JEAn, Robert de (1975). « Préface », dans Green, Julien. Euvres complètes. Paris : Gallimard. T. IV.

SAIz-CERREDA, Maria Pilar (2010). «Henri Thomas : écriture de guerre et silence créateur ou les sources de son identité narrative », dans Çédille. Revista de Estudios Franceses. Abril de 2010, nº 6, p. 229-245.

SimOnET-TEnANT, Françoise (2004). Le journal intime : genre littéraire et écriture ordinaire. Paris : Téraèdre.

STEEL, James (1991). Littératures de l'ombre. Paris : Presses de la Fondation Nationale des Sciences Politiques.

Thomas, Henri (2008). Carnets 1934-1948. « Si tu ne désensables pas ta vie chaque jour... ». Paris Éditions Claire Paulhan.

WERTH, Léon (1992). Déposition. Journal de guerre. 1940-1944. Paris : Viviane Hamy. 